\title{
A Reliable and Efficient Broadcasting Scheme for Mobile Ad Hoc Networks
}

\author{
Kewang ZHANG ${ }^{\dagger a)}$, Student Member and Deyun ZHANG ${ }^{\dagger}$, Nonmember
}

\begin{abstract}
SUMMARY This letter proposes a busy-tone based scheme for reliable and efficient broadcasting in mobile ad hoc networks. Control packets such as RTS, CTS and ACK are ignored in the broadcast scheme, and two busy tones are used, one for channel reservation and the other for negative acknowledgement. Unlike traditional schemes for reliable broadcasting, the proposed scheme is highly efficient as it achieves both collision avoidance and fast packet loss recovery. Simulation results are presented which show the effectiveness of the proposed scheme.

key words: mobile ad hoc networks, media access control, reliable broadcasting, busy tone, hidden terminal problem
\end{abstract}

\section{Introduction}

Broadcasting at the Medium Access Control (MAC) layer is one of the most important services required by higher layer protocols in mobile ad hoc networks (MANET). The current IEEE 802.11 DCF protocol does not offer any MAC layer recovery on broadcast packets. Consequently, unexpected lost packets may deteriorate the quality of broadcast services. To provide reliable broadcasting, many schemes have been proposed recently. However, most of those schemes use control packets, which are neither reliable nor efficient because the control packets themselves are prone to collision and the overheads introduced by such control packets are heavy. Obtaining reliable broadcasting efficiently is a challenging task.

Collision avoidance and packet loss recovery are two fundamental functions for reliable packets delivery. As packets collisions are mainly rooted from hidden terminal (HT) problem in MANET, Ready-To-Send (RTS)/ClearTo-Send (CTS) handshakes are widely used for collision avoidance. Meanwhile, due to error-prone wireless channel, Automatic Repeat Request (ARQ) by using ACK packet is always adopted for packet loss recovery. As RTS/CTS/DATA/ACK handshakes achieve both collision avoidance and packet loss recovery in unicasting scenario, many schemes implement such handshakes in broadcasting. BMW [1] treats broadcasting as multiple unicast operations. For every broadcast data packet, the sender unicasts it to each neighbor using RTS/CTS/DATA/ACK exchange, as is used in unicasting. This scheme is reliable but has severely low efficiency. BMMM [2] tries to improve the efficiency

Manuscript received February 25, 2009.

Manuscript revised April 1, 2009.

${ }^{\dagger}$ The authors are with the School of Electronic and Information, Xi' an Jiaotong University, Xi' an, China.

a) E-mail: zhangkw@xanet.edu.cn

DOI: $10.1587 /$ transinf.E92.D.1470 of BMW by reducing control overheads. This scheme requires broadcast node to exchange RTS/CTS with each intended receiver. When the multiple RTS/CTS exchanges have finished, the pending DATA frame is broadcasted. After that, using a new control frame request for ACK (RAK), the ACKs from the receivers to the sender are also coordinated. Many other schemes aiming at reduce control overheads for reliable broadcasting are presented in [3]-[5].

There are some drawbacks in control-packet based schemes. First, those schemes use RTS/CTS to prevent HTs from interfering with the reception of broadcast packets. However, as RTS/CTS handshakes can not eliminate all HTs in MANET [6] and control packets themselves are prone to collisions, the performance of such schemes degrades dramatically under heavy load conditions [7]. Second, because the broadcast node needs ACKs or CTSs from all receivers, the control overheads are high in those schemes, especially under high nodes density scenarios.

To provide reliable and efficient broadcast service, many people try to improve performance of control-packet based scheme by using busy tones. A busy tone is a signal transmitted over a narrow bandwidth channel with enough spectral separation from the data channel. With a narrow bandwidth, a busy tone can only be sensed as being present or non-present. The busy tone mechanism has been used in several research efforts such as [8]-[10], and [11] to address HT problem in unicasting scenario. BPBT [12] employs the busy tones to provide extra protection for RTS, CTS, ACK and DATA packets. The transmitters turn on the transmit busy tone while sending RTS or data packets. The receivers send the receive busy tone while sending CTS, ACK, or receiving DATA packet. RMAC [13] uses two busy tones named $R B T$ and $A B T$ for HT prevention and positive acknowledgement, respectively. The broadcast node transmits MRTS packet before DATA packet. MRTS packets includes MAC addresses of receivers. All receivers transmit $R B T$ while receiving DATA packets to prevent HTs from transmitting. After receiving DATA packets successfully, each receiver transmits $A B T$ at a given period assigned by the broadcast node. The broadcast node senses these $A B T s$ to confirm that the packets are received successfully by all receivers. The introduction of busy tones improved the efficiency of broadcasting by reducing collisions and decreasing control packets. However, these schemes still rely on control packets. Drawbacks of control-packet based schemes are not overcome in these schemes.

Furthermore, under ARQ mechanism used by previ- 
ous schemes, the broadcast node starts retransmission if it does not receive acknowledgement from one or more receivers. This is based on the assumption that a node in the network has knowledge of all its neighbors. But as we know, the most typical character of MANET is the rapid topology changes because of mobile nodes. Neighbors may leave and join at any time. It is not reasonable to assume that nodes in the network have up-to-date neighbors information for broadcasting. Actually, in most cases, broadcasting itself serves as an approach to exchange topology information among adjacent nodes. Under such schemes, newly joined nodes may not receive broadcast packets reliably because the broadcast node may not be aware of them. Meanwhile, the left or temporal disable of neighbors may lead to poor performance because the broadcast node continues retransmitting packets to it until timeout.

In this letter, a scheme named REB (Reliable Efficient Broadcasting) is proposed. Control packets are completely ignored in REB and two busy tones are used. One busy tone is used for channel reservation, which can prevent HTs from interfering with the broadcast packets. While another busy tone is used for fast negative acknowledgement. As far as the author's knowledge, this is the first attempt to achieve both collision avoidance and packet loss recovery for reliable MAC layer broadcasting by using busy tones only. REB eliminates HTs and achieves packet acknowledgement without introducing control overheads, which is reliable and efficient. And further, no knowledge of neighbors' information are required in REB scheme, which is more suitable for MANET.

\section{Hidden Terminal Problem for Broadcasting}

In wireless networks, the signal to noise plus interference ratio (SINR) must be greater than some threshold $\beta$ and the received signal power must be greater than another threshold $P_{R}$ (namely receive sensitive) for the receiver to detect the received signal correctly.

$$
\left\{\begin{array}{r}
S I N R=10 \log \frac{P_{r}}{\phi+N_{0}} \geq \beta \\
P_{r} \geq P_{R}
\end{array}\right.
$$

Where $\phi$ is accumulated interference and $N_{0}$ is the environmental noise. The received signal power $P_{r}$ :

$$
P_{r}=G \frac{P_{t}}{d^{\lambda}}
$$

Where $P_{t}$ is transmission power of the transmitter, $d$ is the distance between transmitter and receiver (T-R distance for short), $G$ is a constant related to characters of wireless transceiver and antenna, and $\lambda$ is the power loss exponent. In the following discussion, we assume all nodes use a fixed transmission power $P_{T}$.

Three radio ranges namely maximum transmission range $\left(R_{t x}\right)$, interference range $\left(R_{i}\right)$ and carrier sensing range $\left(R_{c s}\right)$ are very important to analyze HT problem.

By combining Eq. (1) and Eq. (2), $R_{t x}$ can be calculated

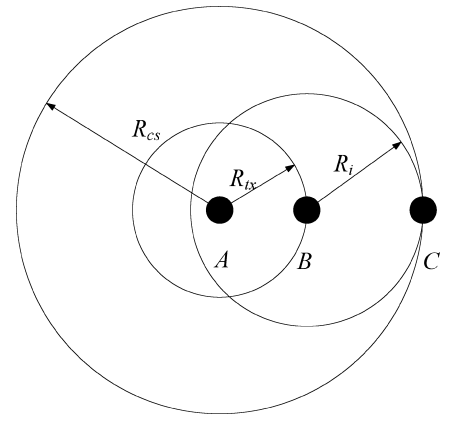

Fig. 1 Hidden terminal problem for broadcasting.

by:

$$
R_{t x}=\left(\frac{G \times P_{T}}{P_{R}}\right)^{\frac{1}{\lambda}}
$$

Supposing node $A$ is the transmitter, node $B$ is the receiver and node $C$ is an interference source, $R_{i}$ is:

$$
R_{i} \geq d \times \beta^{\prime \frac{1}{\lambda}}
$$

Where $\beta=10 \log \beta^{\prime}$. Which can also be calculated from Eq. (1) and Eq. (2) by ignoring $N_{0}$ (This is reasonable as the interference power from node $C$ is much stronger than $N_{0}$ ).

Supposing the transmission power of node $a$ is $P_{t}$ and the Clear Channel Assessment (CCA) threshold is $P_{s}, R_{c s}$ can be expressed as:

$$
R_{c s}=\left(\frac{G \times P_{t}}{P_{s}}\right)^{\frac{1}{\lambda}}
$$

Which can also be induced from Eq. (2). If the received signal power is greater than $P_{s}$, it is said that the channel is busy. Otherwise, idle.

In unicast scenario, as there is only one receiver, HTs are distributed around the receiver, and $R_{i}$ is mainly affected by $d$. But in broadcast scenario, all nodes within $R_{t x}$ of broadcast node are all receivers. Therefore, HTs are distributed around the broadcast node, which is different from unicast. From Eq. (4) we can find that the $R_{i}=R_{t x} \times \beta^{\prime \frac{1}{\lambda}}$ when $d=\mathrm{R}_{t x}$. So the HTs for broadcasting are distributed within $R_{t x}+R_{t x} \times \beta^{\prime \frac{1}{\lambda}}$ around the broadcast node (shown in Fig. 1). If a busy tone is used by broadcast node to eliminate HTs, by adjusting busy tone CCA threshold in Eq. (5), $R_{c s}$ of busy tone can be adjusted to $R_{t x}+R_{t x} \times \beta^{\prime \frac{1}{\lambda}}$ and covers all HTs. Our proposed scheme is based on this finding.

\section{Description of REB Scheme}

Two out of band busy tones are used in REB scheme, which are also used in DBTMA [14] and many other MAC protocols for efficient unicasting. As busy tones and data channel use different frequency with enough separation, interference among them can be ignored. As illustrated in Fig. 2, one busy tone named $B T b$ is used for channel reservation to eliminate HTs, and another busy tone named BTa is used for 


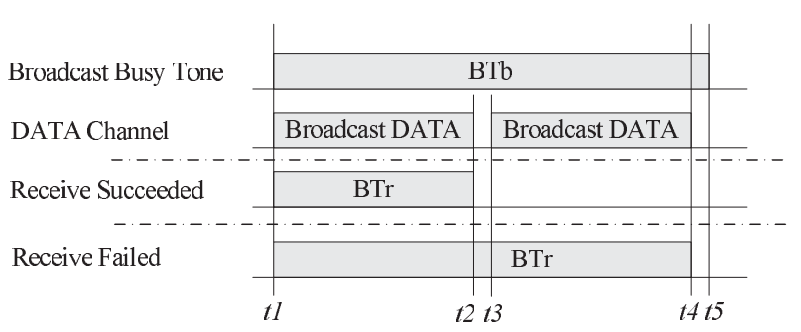

Fig. 2 Reliable broadcasting in REB scheme.

negative acknowledgement.

Before broadcasting, the broadcast node senses $B T b$ and data channel. If either $B T b$ or data channel is sensed busy or both are busy, there must be a unicasting or broadcasting. The broadcast node defers its transmission and retries after these two channels become idle again. If both channels are sensed idle, the broadcast node starts $B T b$ and transmits broadcast packets in data channel. All nodes that sensed $B T b$ are prevented from transmission (including unicast packets and broadcast packets).

On sensing $B T b$, all receivers start sending $B T a$ and begin to receive in data channel. The receiver stops $B T a$ if received the broadcast packet successfully, continues $B T a$ otherwise. The broadcast node senses $B T a$ after transmitting the packet. If $B T a$ is sensed, there must be at least one receivers have not received the packet correctly. Thus, the broadcast node continues retransmitting until no BTa exists.

Please note that HTs and receivers are all covered by $B T b$, but they need different process (HTs need only defer their transmission, while receivers must receive the broadcast packet), how to distinguish HTs from receivers is a critical problem. A simple approach is used in REB. Nodes covered by $B T b$ sense received signal power of $B T b$. If the received signal power of $B T b$ is higher than $P_{R}$, the node must be a receiver. While the node must be a HT if the received signal power is between $P_{R}$ and $P_{s}$.

The efficiency of REB lies in four facts. First, no control packets and only two busy tones are used in REB scheme. As busy tones are collisions free [14], REB can improve the performance of broadcasting dramatically, especially under heavy traffic load condition. Second, BTb reserves channel and prevents all HTs from transmitting. As no collisions introduced by HTs occur, REB is more efficient as compared to traditional schemes where RTS/CTS handshakes can only control HTs within $\mathrm{R}_{t x}$. Third, REB require no knowledge of neighbors information, which is more suitable for MANET. And finally, the overheads for acknowledgement are much lower as compared to control packets based approaches. As no collision among busy tones, the broadcast node can senses $B T a$ successfully even if there are more than one receivers sending $B T a$ simultaneously.

\section{Simulation Results}

The performance of the proposed scheme is evaluated by

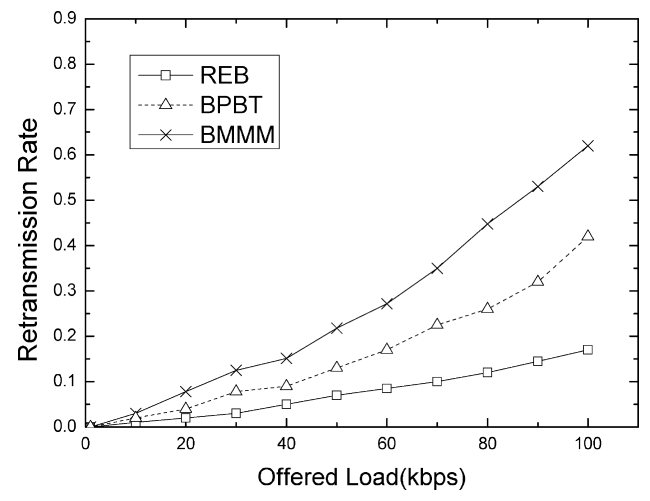

Fig. 3 Retransmission rate of broadcast. $D b=10 \mathrm{pkt} / \mathrm{s}$.

extensive simulations using GloMoSim [15]. For compare purpose, BMMM, BPBT and REB are implemented in the simulator. 802.11 DCF is used for unicast in all simulations. The data rate of wireless device is set to $2 \mathrm{Mbps}$ and no rateadaptation is used.

The simulation configurations are as follow. 64 nodes are distributed randomly in an area of 1000 by 1000 meters. The random way point mobile model is used for nodes mobility and the speed of the mobile nodes is $15 \mathrm{~m} / \mathrm{s}$. The unicast traffic model is CBR (Constant Bit Rate) and the deliver rate is $D u$ packets per second. 8 unicast traffic flows are configured in the simulations. The source nodes and destination nodes are selected randomly at initialization time. Every nodes in the network broadcast packets with deliver rate $D b$. The length of unicast and broadcast packets are both set to 256 Byte.

The first simulation is conducted to evaluate the advantage of channel reservation by using BTb. The traffic load includes unicast and broadcast packets. The broadcast delivery rate $D b$ is set to 10 packets per second. Figure 3 shows the broadcast retransmission rate against offered unicast traffic load. As all HTs in REB are well controlled by channel reservation using $B T b$ and collisions introduced by HTs are minimized, the broadcast retransmission rate of REB is much lower than that of BMMM while traffic load becomes heavy. The BPBT scheme uses busy tone to eliminate HT problem too, but the busy tone is transmitted by the receiver rather than the broadcast node. As control packets themselves are prone to collisions, some of the receivers can not receive RTS packet correctly, which may leads to unsuccessful DATA packets reception. Therefore, such busy tone is not as effective as $B T b$ used by REB scheme, and the broadcast retransmission rate is much higher than that of REB.

To further investigate the overall performance of REB scheme, another simulation is conducted. The simulation configurations are very similar to the first simulation except that the successful delivery rate is evaluated rather than the retransmission rate. The maximum retry count of broadcast packets is set to 4 , which is the default configuration of the simulator. Figure 4 shows the broadcast packets successful delivery rate against offered unicast traffic load. In BMMM, 


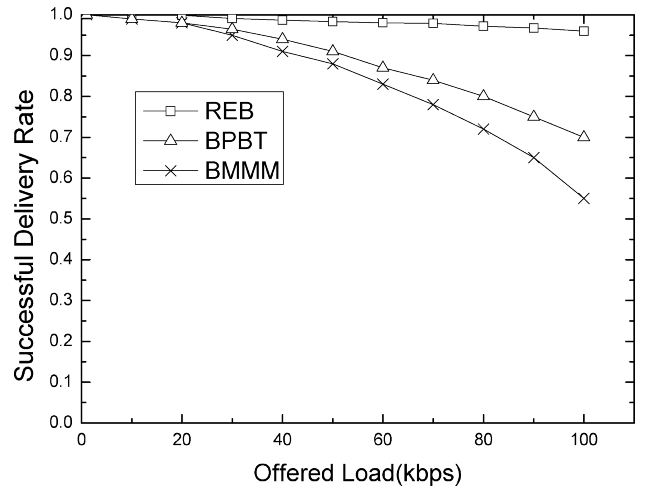

Fig. 4 Successful delivery ratio of broadcast.

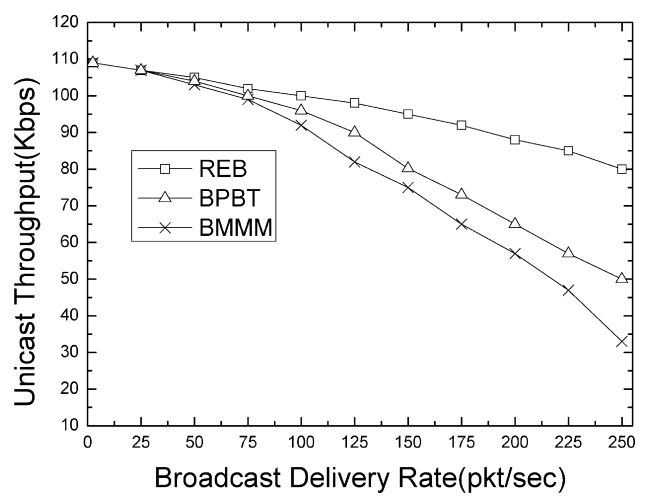

Fig. 5 Average node throughput (unicast only).

the successful delivery rate decreases dramatically as the traffic load becomes heavy. The reasons lies in two facts. First, control packets are prone to collisions under heavy traffic load condition. Second, RTS/CTS used in BMMM can not eliminate HTs, which leads to frequent collision when traffic load gets heavy. In BPBT, busy tone is used to eliminate HTs of DATA packets, which is more effective than RTS/CTS handshakes. And the successful delivery rate is higher than BMMM when traffic load is heavy. While in REB scheme, as no control packets are used for broadcast and all HTs are eliminated by channel reservation, the broadcast delivery rate keeps high even if the traffic load gets heavy.

To evaluate the impact of broadcast on network performance, the third simulation is conducted. In this simulation, each node always has unicast packets to be sent, which means a saturated traffic scenario. And the offered broadcast traffic $D b$ is variable. Figure 5 shows average node throughput against broadcast delivery rate. In BMMM scheme, with the increase of offered broadcast load, the average node throughput degrades to $30 \%$ of the maximum throughput where broadcast delivery rate reaches $250 \mathrm{pkt} / \mathrm{sec}$. The main reasons are that under heavy load condition, the control packets are prone to collision and packets loss recovery scheme introduces great control overheads. The channel is wasted in retransmitting the broadcast packets and packet acknowledgements, which leads to lower node throughput.
BPBT performs better than BMMM because most HTs of DATA packets are eliminated by busy tones. While in REB scheme, such drawbacks are overcame by busy tones without any control packets.

\section{Conclusion}

This letter proposes a new scheme for reliable broadcasting in MANET. With two busy tones, the proposed scheme achieves both collision avoidance and packet loss recovery. The simulation results show that the proposed scheme is highly reliable and efficient as compared with existing broadcasting schemes using traditional control frames.

Our proposed scheme needs two busy tones for reliable and efficient broadcasting. As busy tone channel is a simplified wireless channel which carries no information, dual busy tone channels can be implemented by using two different channels with enough frequency separation from each other and DATA channel. As extra hardware is required by REB, the total cost of the node is increased (less than $\$ 10$ even if two separate wireless transceivers such as CC1000 [16] are used for busy tones). But we believe that the gain of this scheme in network performance is a good incentive to add such hardware to network nodes.

\section{References}

[1] K. Tang and M. Gerla, "MAC reliable broadcast in ad hoc networks," Proc. IEEE Military Communications Conference 2001, pp.10081013, 2001.

[2] S. Min-Te, H. Lifei, A. Arora, and L. Ten-Hwang, "Reliable MAC layer multicast in IEEE 802.11 wireless networks," Proc. IEEE ICPP 2002, pp.527-536, 2002.

[3] S. Shiann-Tsong, T. Yihjia, and C. Jenhui, "A highly reliable broadcast scheme for IEEE 802.11 multi-hop ad hoc networks," Proc. IEEE International Conference on Communications 2002, pp.610$615,2002$.

[4] L. Ki-Ho and C. Dong-Ho, "A multiple access collision avoidance protocol for multicast services in mobile ad hoc networks," IEEE Commun. Lett., vol.7, no.10, pp.508-510, 2003.

[5] J. Xie, A. Das, S. Nandi, and A.K. Gupta, "Improving the reliability of IEEE 802.11 broadcast scheme for multicasting in mobile ad hoc networks," IEE Proc., Commun., vol.153, pp.207-212, 2006.

[6] X. Kaixin, M. Gerla, and B. Sang, "How effective is the IEEE 802.11 RTS/CTS handshake in ad hoc networks," Proc. IEEE GLOBECOM 2002, pp.72-76, Taipei, 2002.

[7] D. Towsley, J. Kurose, and S. Pingali, "A comparison of senderinitiated and receiver-initiated reliable multicast protocols," IEEE J. Sel. Areas Commun., vol.15, pp.398-406, 1997.

[8] F. Tobagi and L. Kleinrock, "Packet switching in radio channels: Part II-The hidden terminal problem in carrier sense multiple-access and the busy tone solution," IEEE Trans. Commun., vol.23, no.12, pp.1417-1433, 1975.

[9] Z. Hongqiang, W. Jianfeng, and F. Yuguang, "DUCHA: A new dualchannel MAC protocol for multihop ad hoc networks," IEEE Trans. Wireless Commun., vol.5, no.11, pp.3224-3233, 2006.

[10] W. Ping, J. Hai, and Z. Weihua, "A new MAC scheme supporting voice/data traffic in wireless ad hoc networks," IEEE Trans. Mobile Comput., vol.7, no.12, pp.1491-1503, 2008.

[11] W. Shih-Lin, T. Yu-Chee, and S. Jang-Ping, "Intelligent medium access for mobile ad hoc networks with busy tones and power control," IEEE J. Sel. Areas Commun., vol.18, no.9, pp.1647-1657, 2000. 
[12] C. Chun-Yuan, E.H.K. Wu, and C. Gen-Huey, "A reliable and efficient MAC layer broadcast protocol for mobile ad hoc networks," IEEE Trans. Veh. Technol., vol.56, no.4, pp.2296-2305, 2007.

[13] W.-S. Si and C.-Z. Li, "RMAC: A reliable MAC protocol supporting multicast for wireless ad hoc networks," J. Computer Science and Technology, vol.20, pp.702-712, 2005.

[14] Z.J. Haas and J. Deng, "Dual busy tone multiple access (DBTMA)-a multiple access control scheme for ad hoc networks," IEEE Trans.
Commun., vol.50, no.6, pp.975-985, 2002.

[15] X. Zeng, R. Bagrodia, and M. Gerla, "GloMoSim: A library for parallel simulation of large-scale wireless networks," Twelfth Workshop on Parallel and Distributed Simulation, pp.154-161, Banff, 1998.

[16] Texas Instruments, "Single-Chip Very Low Power RF Transceiver," available at http://focus.ti.com/lit/ds/swrs048a/swrs048a.pdf 